\title{
Application of Hypothetical Quantum Scattering Model for the Design of Novel Electroluminescence Device
}

\author{
Hyo Weon Jang \\ Department of Chemistry, Sunchon National University, Sunchon, Chonnam 540-742, Korea \\ Received August 28, 2001
}

\begin{abstract}
We present a hypothetical quantum scattering model to propose a novel electroluminescence device. Adopting the features of solid state semiconductor LED and exciplex laser, the cathode (electron incoming potential) and anode (electron outgoing potential) are made to correspond to two 1-dimensional resonance supporting potentials, and the light emitting part to an interaction potential in the intermediate region. When an extenal voltage is applied, the electron flows into the cathode having small work function. Subsequently it flows via LUMO of the "electron incoming potential", loses kinetic energy emitting a photon, then continues to flow via LUMO of the "electron outgoing potential" unlike the conventional LUMO to HOMO transitions occurring in solid state semiconductor LED. In this model, the photon frequency can be controlled by adjusting the applied voltage. The model hopefully could be realized as partially conjugated hydrocarbon chains.
\end{abstract}

Keywords : Quantum, Model, Electroluminescence, Simulation.

\section{Introduction}

The light emitting device has important practical applications such as in multimedia display devices. It converts electrical energy into light of suitable color and is called electroluminescence (EL) device. One of the virtue that a good device must have is superior energy conversion efficiency, since efficient device saves electrical energy, and lasts long than otherwise. Meanwhile, less efficient device produces large amount of heat which shortens the device life. Ease of fabrication, cheap production cost, emission of various colors and flexibility of the device would also be additional virtue. ${ }^{1}$

Inorganic and organic materials have been used as light emitting media. The former includes solid state semiconductors, and the latter includes small molecular fluorescent dyes and conjugated polymers. Particularly, the organic polymers have several advantages over other materials such as low driving voltage, convenient fabrication procedure, cheap cost, possibility of making flexible display device and making the emitting cell size large. ${ }^{1}$

However, they operate with relatively low conversion efficiencies of a few percent, typically, at present state. The implication of such drawbacks includes energy waste, and more importantly shortening of the device life caused by the heat produced by nonradiative current. ${ }^{1}$ Therefore, the improvement of energy conversion efticiency of the EL device is valuable.

One of the origin of such low efficiency could be traced back to the randomness of the microscopic structure which may increase nonradiative quenching of electron energy, whereas the device itself is operating macroscopically. Therefore, it may be fruitful to start the design of novel device from microscopic level at which we may exclude any

E-mail: hyo(âsunchon.ac.kr randomness of the device structure.

Hoping for developing such highly efficient EL device, we propose a simple hpothetical quantum scattering model. The model effectively represents a microscopic system composed of two segments of conducting molecular wires separated by low-conductivity molecular bridge. If we adopt suitable parameters to define the necessary 1-dimensional potential energy surfaces (PES's) corresponding to the model, we can perform the quantum scattering simulation where the raw numerical results are the transmission probabilities of electron from one side of the system to the other side via various pathways such as radiative and nonradiative transmissions. Then, we may interpret the numerical results in terms of the performance of such hypothetical EL device, and learn a hint which may be helpful for designing a realistic novel device.

The present model adopts several conceptual components from the well-known light emitting apparatuses like light emitting p-n junction diode (LED), and exciplex laser. ${ }^{2.3} \mathrm{We}$ discuss the details in later section.

In Sec. 2, we detail how the electron energy converts to light in our hypothetical model. In the following Sec. 3, we present sample calculation results and interpret them in terms of the electron-to-photon conversion efficiency. Also, the dependence of efficiency on the molecular structural characteristics is discussed. In the final Sec. 4 , we speculate how an EL device corresponding to our model could be made in real life. And, finally, we discuss what further studies could be done based on the present work.

\section{Present Model System}

We explain how our model system is constructed below (see Figure 1).

We intend to utilize spontaneous emission phenomenon for light emitting mechanism, where an electron occupying 


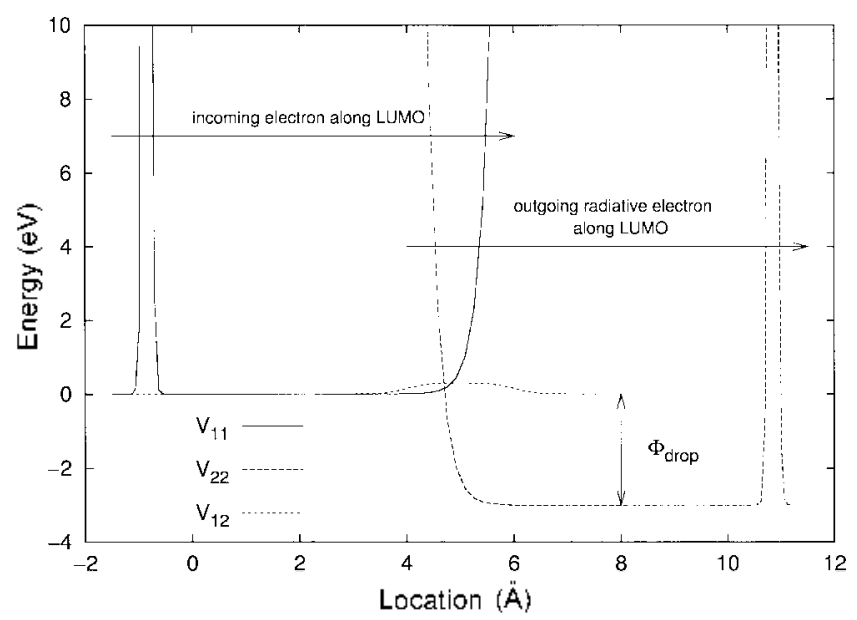

Figure 1. PES's of the present model. $F_{11}$ and $F_{22}=V_{33}$ supports resonance states with energy levels of $0.8,3.1,7.0,12.3 \mathrm{eV}$, and so on, respectively. The incoming electron is chosen to have kinetic energy of $7 \mathrm{eV}, F_{12}=F_{13}$ is adjusted to split the energy levels by 0.1 to $0.2 \mathrm{eV}$. The third level from the ground is chosen as LUMO in the calculation and indicated by incoming and outgoing waves denoted by long arrows. The potential drop is denoted by $\Phi_{\text {ilvof }}$.

higher energy level state emits light spontaneously and makes transition to unoccupied lower energy level state. ${ }^{4}$ Higher energy electrons are supplied continuously by applying external voltage which also maintains the energy level separation between the higher and lower energy states involved in the radiative transition.

The higher energy electron occupies a resonance state supported by an appropriate 1-dimensional PES (namely, $V_{11}$ ) and is represented by a unit probability flux incoming wavefunction from the cathode. The lower energy electron will occupy a similar resonance state, supported by another 1-dimensional PES $\left(V_{22}\right)$, represented by outgoing wavefunction toward the electron extraction electrode (anode) after "radiative transmission" (equivalent to "spontaneous emission in our model").

The radiative transmission probability depends on the interaction potential $\left(V_{12}\right)$ which represents the coupling between outgoing and incoming wavefunctions. With only above three PES's, we cannot represent the nonradiative transmission which inevitably occurs in realistic device in the form of ohmic current loss and largely contributes to the inefficient performance of an EL device. To account for this effect, we choose to include additional 1-dimensional PES $\left(V_{33}\right)$ and interaction potential $\left(V_{13}\right)$. The $V_{33}$ supports another outgoing wavefunction which accommodate the nonradiatively transmitted electron, and $V_{13}$ determines the nonradiative transmission probability. In terms of scattering theory, our model comprises three open channels, i.e., an incoming scattering state of an electron from the cathode, an outgoing scattering state resulted from radiative transmission, and another outgoing scattering state resulted from nonradiative transmission.

In the calculation, we assume that the effect of the applied external voltage occurs only in the interaction region where the interaction potentials couple $V_{11}, V_{23}$, and $V_{33}$. In other words, it is assumed that the electrostatic potential drop ( $\Phi_{\text {shop }}$ ) only appears in the region where $V_{11}$ and $V_{22}$ (or $V_{33}$ ) overlaps with each other, and that the shapes of the PES's don't change (only the base of $V_{11}$ is raised by the value of $\Phi_{\text {thop }}$ against $V_{22}$ (or $V_{\text {za }}$ )).

The above assumption regarding the position of potential drop may be rationalized by noting that the conductivity of the wires corresponding to $V_{11}, V_{22}$ (or $V_{31}$ ) should be inuch greater than that of the bridge corresponding to $V_{12}$ (or $V_{13}$ ).

\section{Calculation and Interpretation}

We use the log derivative version of Koln variational method of Manolopoulos and Wyatt to obtain numerical transmission probabilities for our model. "This method has several convenient features such as producing unitary scattering matrix, and use of real algebra only, and rapid convergence as basis set grows. Especially, it is suitable for 1-dimensional problems like the present model, because one set of basis function is sufficient, not multiple sets on different coordinate systerns.

We first adjust the parameters for $V_{11}$ such that it supports several resonance states with energy levels separated by a few electron volts $(\mathrm{eV})$. The shapes of $V_{22}$ and $V_{3 z}$ are chosen to be identical with $V_{11}$ except that it is transposed around the center of the system, and $V_{33}$ appears to superimpose with $V_{22}$ regardless whether an external voltage is applied or not. The potentials are translated against each other so that the incoming and outgoing scattering wavefunctions on each PES have substantial overlaps with each other. We call this overlap region as interaction region where the interaction PES's ( $V_{12}$ and $V_{13}$ ) act. The magnitude of the interaction PES's are adjusted to split the energy levels a few tenths eV when $V_{11}$ and $V_{22}$ (or $V_{33}$ ) are coupled. We test several values for the range and magnitude of interaction PES's to determine the optimal condition for better efficiency of EL.

We arbitrary choose the third level as the LUMO which corresponds to the relevant incoming and outgoing waves.

In Figure 2, we present the transmission probabilities as a function of emitted photon energy when $\Phi_{d r o p}$ is chosen to be $2 \mathrm{eV}$ and $3 \mathrm{eV}$ (which covers from red to blue visible light range). This spectrum may be interpreted in a similar manner as the case of the exciplex laser where the emitted photon frequency is detemined by the largest probability transition. ${ }^{3}$ Therefore, it is noted that the emitted photon has the same energy as $\Phi_{\text {irop. }}$. This observation allows us a way to control the emitting light color of EL device by simply changing the applied voltage. However, since the visible light range covers approximately from $2 \mathrm{eV}$ to $3 \mathrm{eV}$, the energy level spacing above and below the LUMO should be greater than about $3 \mathrm{eV}$, such condition is satisfied for the present parameters. This control is more or less not possible in the conventional EL device where the light color is determined by the bandgap of the light emitting material, consequently, the light color is independent of the applied voltage. $^{1}$ 


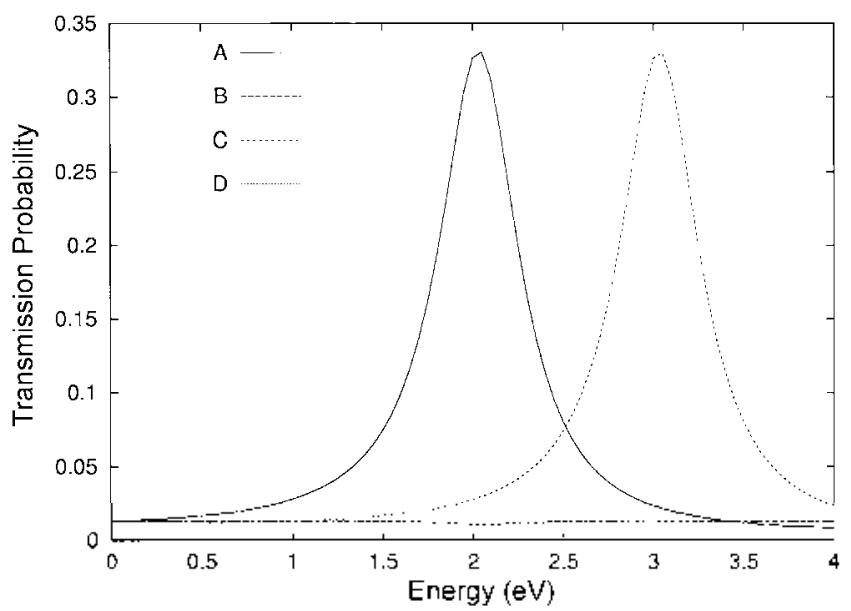

Figure 2. Transmission probabilities of electron via radiative ("A", " $\mathrm{C}$ ") and nonradiative ("B", "D") channels. The incoming electron has kinetic energy of $7 \mathrm{eV}$ corresponding to the third resonance state from the ground of $V_{11}$. "A", "B" use $2 \mathrm{eV}$ and "C", "D" use 3 $\mathrm{eV}$ as $\Phi_{\text {drog. }}$

Regarding the conversion efficiency, the ratio of the emitted photons over the net transmitted electrons in the Figure 2, is greater than $96 \%$ at the peak. The major reason behind this large ratio is that the nonradiative outgoing wave does not couple effectively with any resonance state, while radiative outgoing wave does so with a resonance state (LUMO). Although we use $V_{12}=V_{1:}$ for the results in Figure 2 , the conversion efficiency directly depends on the relative ratio of the $V_{12}$ over $V_{13}$ which corresponds to the interaction strength of radiative and nonradiative transmission, respectively. It is observed that, as the magnitude of $V_{12}$ surpasses that of $V_{13}$, the conversion efficiency increases, although we don't present numerical results here.

The absolute values of transmission probabilities determine the overall current density in the sense that small values indicate weak EL and, also small ohmic current per device unit. Also the assumption mentioned above about $\Phi_{\text {drop }}$ is justified in this case because the conductivity of the bridge (interaction region) is minimized. The electrostatic potential drop is larger where the conductivity is small. In other words, as the overall transmission probability increases, the light intensity and ohmic current also increase, however, the validity of the model reduces.

The effect of different interaction region parameters can be seen in Figure 3. We note that the largest transmission occurs when the overlap between two LUMO's are favorable such as in the case of " $\mathrm{B}$ " where the LUMO's wavefunction lobes are in phase: while they are not as in the cases of " $\mathrm{A}$ " and " $\mathrm{C}$ " in Figure 3. Therefore, if the interaction region is adjusted so that the phases of incoming and outgoing wavefunction are in phase, the transmission probability should be larger than the out of phase cases. This means that the light intensity is larger per device unit as in the case " $B$ " than otherwise.

We may find the analogy of the present model with other existing light emitting devices. Analogy with exciplex laser may be found as follows. The incoming wave (LUMO)

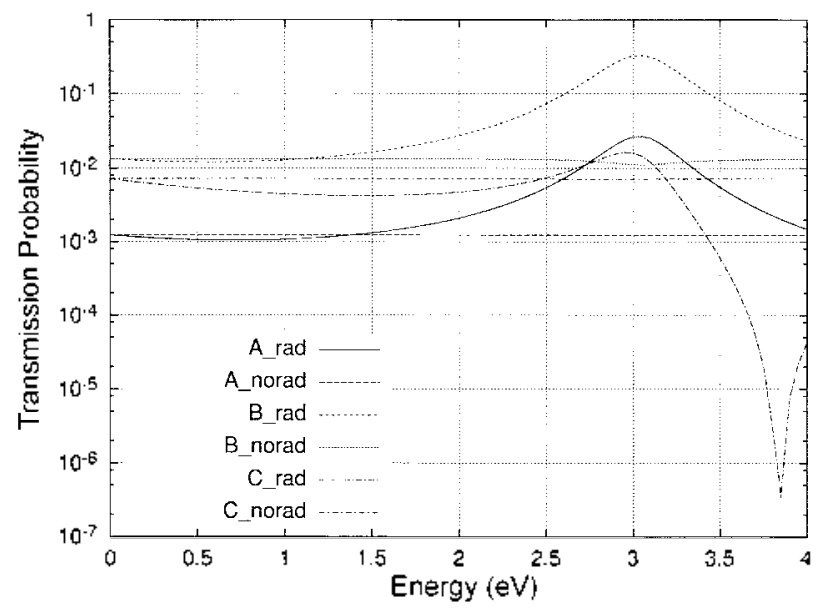

Figure 3. Effect of the interaction length on transmission probabilities. The lengths of interaction regions increase in the order of "A", "B", "C". The suffix "rad" and "norad" means radiative and nouradiative transmission, respectively. We use same parameters as in Figure 2. $\Phi_{\text {drop }}$ is chosen to be $3 \mathrm{eV}$. Note that results " $\mathrm{B}$ " also appear in Figure 2 and is the most favorable.

supported by the higher potential energy corresponds to the resonance state supported by electronic excited state and the radiative outgoing wave supported by the lower potential energy corresponds to the Franck-Condon wave supported by electronic ground state in exciplex laser, respectively. The role of interaction PES $V_{12}$ is in parallel with the spontaneous and/or stimulus emission rates which involve the square modulus of transition dipole moment between the two relevant states. ${ }^{3}$

In comparison with solid p-n junction semiconductor LED, we should note the difference first. In the conventional LED, the radiative transmission of electron occurs from the conduction band (corresponding to LUMO) of the cathode side to the valence band (corresponding to HOMO) of the anode side, ${ }^{2}$ while in our model the transmission is between LUMO and LUMO. The difference originates from the fact that in our model the electrons occupy nondegenerate (or a band with extremely narrow bandwidth) electronic state, while in the conventional LED the electrons can occupy a wide range of energy levels in a band of states. On the other hand, it is similar to our model that the emitted photon energy is detemined by the potential drop in the interface region (interaction region in our model). In our model, the upper bound of the emitted photon energy is the HOMOLUMO energy gap.

\section{Conclusions}

In this section, we will discuss several points to consider for constructing a real EL device and several hypothetical EL device models based on the present simulation results. Finally, we note a few directions of further studies.

To reduce the device driving voltage, which is equivalent to saying that the applied voltage should be as small as the electrostatic potential drop in the interaction region, we should use "small work function metal" for the cathode. 
While, for the anode, it may be necessary to avoid "large work function metal" since such anode may induce the HOMO partially vacant and subsequently make HOMO available for additional spontaneous emission channel. As a result, the emission spectrum and the dynamics may become unnecessarily complicated.

We might imagine that the present model system may represent two segments of completely conducting conjugated polymers joined by limited conducting molecular fragment such as 1,4-cyclohexadiene. To synthesize a molecular wire with large energy level spacing, it might be helpful to substitute appropriate functional groups along the molecular chains.

We also may connect duplicates of the above models (considering it a unit EL cell device) in a series, and make a composite long molecular wire system. When we connect the ends of wire to the electrodes and apply an external voltage, the external voltage should be determined by the potential drop in a unit EL cell times the number of cells in the composite wire, such voltage may be quite large for a very big composite system. To avoid the need of applying such impracticably large voltage, we might imagine an macroscopic arrangement where the cathode and anode are nailed into the wire alternatively with a sub-macroscopic spacings. In that way, the required external voltage may be dramatically reduced. Also, in this arrangement, the wires may be positioned randomly in a suitable inert matrix material between them. The overall arrangement might look similar to the existing conducting organic polymer EL device where the EL materials are placed in a matrix of thin film between the plates of electrodes. They differ in that the electrodes protrude in a form of needles from the cathode and anode plates in our hypothetical device.

Further studies may be directed to make the model system close to a realistic 3-dimensional one, or to develop a method to reduce 3-dimensional realistic calculation results to the potential parameters used in our model. For example, the magnitudes of interaction potentials may be chosen to reproduce the calculated energy level splittings and the spontaneous emission rate obtained by $a b$ initio quantum mechanical calculation.

In conclusion, we presented a simple hypothetical quantum scattering model which hopefully may help in designing a novel EL device with high efficiency and convenient color control features.

Acknowledgment. This paper was conducted by the research fund provided by Korean Council for University Education, Support for 2000 Domestic Faculty Exchange Program.

\section{References}

1. Salbeck. J. Ber: Bthsenges. Phys. Chen. 1996. 100. 1667.

2. Kittel. C.; Kroemer, H. Theinal Physics. 2nd ed.; Freeman: San Francisco, 1980.

3. Atkins, P. W. Physical Chemistry, 6th ed; Oxford University Press: Oxford, 1998.

4. Weissbluth, M. Atoms and Molecules; Academic Press: New York, 1978 .

5. Manolopoulos, D. E,; Wyatt, R. E. Chem. Phys. Lett. 1988, 152, 23. 